\title{
Quantitative assessment of macular thickness in normal subjects and patients with diabetic retinopathy by scanning retinal thickness analyser
}

\author{
Yusuke Oshima, Kazuyuki Emi, Shigeki Yamanishi, Masanobu Motokura
}

\begin{abstract}
Aims-To evaluate the scanning retinal thickness analyser (RTA), a novel noninvasive imaging instrument, in diagnosing and quantitatively characterising diabetic macular oedema, and to investigate the relation between central macula thickness measured by RTA and other clinical examinations.

Methods-Central macular thickness was measured using the RTA in 40 normal subjects and 60 patients with diabetic retinopathy. The reproducibility of the retinal thickness measurements was evaluated by calculating the mean of the inter- and intrasession variations. Central macular thickness was correlated with the results of visual acuity measurements, biomicroscopy, and fluorescein angiography.
\end{abstract}

Results-Intra- and intersession reproducibility of the RTA in normal subjects was plus or minus $5.2 \%(16 \mu \mathrm{m})$ and plus or minus $6.1 \%(19 \mu \mathrm{m})$, respectively. The mean central macular thickness was 182 (SD 16) $\mu \mathrm{m}$ in normal subjects, 283 (116) $\mu \mathrm{m}$ in diabetic eyes without clinically significant macular oedema (CSMO), and $564(168) \mu \mathrm{m}$ in diabetic eyes with CSMO. Central macular thickness was significantly greater $(p<0.001)$ in eyes with diabetic retinopathy than in normal subjects, even when macular thickening did not meet the standard for CSMO $(p=0.019)$ measured by biomicroscopy. Although greater fluorescein leakage at the macula results in greater central macular thickness, only eyes with diffuse leakage had statistically significant macular thickening compared with normal subjects $(p=0.022)$. Central macular thickness measured with the RTA was significantly correlated with the logarithmic converted visual acuity $\left(r^{2}=0.76\right)$ in diabetic eyes.

Conclusion-Scanning RTA, which has good reproducibility, might be useful to quantitatively detect and monitor macular thickening in diabetic retinopathy. Central macular thickness was highly correlated with logarithmic converted visual acuity in diabetic macular oedema.

(Br F Ophthalmol 1999;83:54-61) Yusuke Oshima, MD Department of

Ophthalmology, Osaka Rosai Hospital, 1179-3,

Nagasone-cho, Sakai-City,

Osaka 591-8025, Japan.

Accepted for publication 26 August 1998

Diabetic macular oedema, a common cause of central visual loss in patients with diabetic retinopathy, ${ }^{1-3}$ usually results from the break- down of the blood-retinal barrier, which leads to abnormal fluid accumulation in the retinal layer and increased retinal thickness. ${ }^{45} \mathrm{Al}-$ though early detection and diagnosis are important to successfully treat diabetic macular oedema, the clinical detection and evaluation methods currently used are limited to slit lamp biomicroscopy, stereoscopic fundus photography, and fluorescein angiography, which provide only a subjective evaluation of retinal thickness. ${ }^{6}$ Although fluorescein leakage from the perifoveal capillary network indicates the breakdown of the blood-retinal barrier at the macula, angiographic findings are neither a reliable nor a quantitative indicator of the degree of fluid accumulation and retinal thickening. ${ }^{78}$ Subjective observations using slit lamp biomicroscopy and/or fundus stereoscopy cannot quantify the subtle changes of macular thickening. ${ }^{9-11}$

Recently, several new techniques to measure quantitatively the retinal thickness have been explored. ${ }^{12-14}$ The scanning retinal thickness analyser (RTA), a novel instrument developed for non-invasive, multiple optical cross sectional retinal visualisation that is based on the principle of laser biomicroscopy, ${ }^{15}$ provides objective and quantitative measurements of retinal thickness. ${ }^{16}$ The distance between the images of the anterior and posterior retinal intersections is calculated by an analysis algorithm to produce accurate retinal thickness mapping. Zeimer and associates ${ }^{16}$ and Asrani and colleagues ${ }^{17}$ in their early experience with the RTA, reported its potential for clinical use. Landau and associates ${ }^{18}$ reported their in vivo measurements of retinal thickness in healthy volunteers. At present, however, few studies of quantitative retinal thickness measurements have been reported in patients with diabetic retinopathy. ${ }^{19-21}$

In this prospective study, we used a commercially available, rapid scanning RTA with a newly developed analysis algorithm to examine the macular thickness in age and race matched groups of normal subjects and patients with diabetic retinopathy. We evaluated the clinical reproducibility of this new instrument and investigated its potential as an objective test to diagnose and quantitatively measure the central macular thickness in diabetic retinopathy.

Materials and methods

SUBJECTS

A prospective study was designed to assess quantitatively retinal thickening in normal subjects and patients with diabetic retinopathy 
Table 1 Demographic data for normal subjects and patients

\begin{tabular}{|c|c|c|}
\hline & $\begin{array}{l}\text { Normal } \\
\text { subjects }\end{array}$ & $\begin{array}{l}\text { Patients with } \\
\text { diabetic } \\
\text { retinopathy }\end{array}$ \\
\hline Group size (no) & 40 & 60 \\
\hline Female/male & $22 / 18$ & $33 / 27$ \\
\hline Age (years) (SD) & $53.2(15.8)$ & $55.7(10.1)$ \\
\hline Range & 25 to 69 & 24 to 73 \\
\hline \multicolumn{3}{|c|}{ Refractive error (D) ${ }^{\star}$} \\
\hline Mean (SD) & $-0.75(1.78)$ & $-0.05(1.34)$ \\
\hline Range & -1.75 to +1.50 & -1.50 to +2.25 \\
\hline \multicolumn{3}{|l|}{ Axial length (mm) } \\
\hline Mean (SD) & $24.21(0.67)$ & $24.55(0.83)$ \\
\hline Range & 22.87 to 25.54 & 23.17 to 25.87 \\
\hline LogMAR (SD) & $0.08(0.52)$ & $3.78(3.61)$ \\
\hline
\end{tabular}

using a scanning RTA. After approval by the human subjects committees of our institution, 60 patients (34 men and 26 women) with diabetes and various degrees of retinopathy were recruited for the study from the retinal section of the Department of Ophthalmology at Osaka Rosai Hospital, Osaka, Japan. All eyes had a best corrected visual acuity of 20/400 or better. No patient had laser treatment (grid or focal) of the posterior pole before participation in the study. Subjects with corneal or lenticular disease, hazy media, or extensive lipid accumulation at the fovea were excluded from the study because the irregular reflections from hard exudates that accumulated in the retina and the interference of the media opacities with the scanning of the laser may preclude a detailed evaluation of the retinal thickness. Patients with macular oedema of non-diabetic aetiology or other retinochoroidal disorders also were excluded.

Forty age matched individuals (22 men and 18 women) volunteered to participate as normal control subjects. The corrected visual acuities of this group ranged from 20/25 to $20 / 20$. With the exception of the corrected visual acuities, all variables (age, sex, refractive error, and race) were well matched in both groups. Normal subjects were excluded if they had a history of diabetic mellitus or other ocular disease except refractive error. The demographic data of the study participants are shown in Table 1.

In the normal subjects, one eye of each volunteer was randomly selected for retinal thickness measurement. In patients with diabetic retinopathy, the study eye also was randomly selected to assure the independence of data analysis when both eyes met the criteria. Before the study, a full explanation of the procedure was given to all participants and informed consent was obtained in all cases.

PROCEDURES

The examination included the following assessments: corneal refractive power, refractive error, and best corrected visual acuity; a clinical examination; fundus photography; fluorescein angiography (patient group only); and retinal thickness measurement. A detailed fundus examination was performed by slit lamp biomicroscopy with a 90 dioptre lens or Goldmann contact lens, fundus photography, fluorescein angiography using a 35 degree field fundus camera, and retinal thickness measurement using a RTA was performed in all patients with diabetic retinopathy.

The clinical diagnosis of diabetic retinopathy was based on the results of a detailed slit lamp biomicroscopic examination and fluorescein angiography. The severity of diabetic retinopathy was classified by means of a fundus examination and photography according to the criteria of the Early Treatment Diabetic Retinopathy Study (ETDRS) ${ }^{22}$ - that is, nonproliferative diabetic retinopathy (levels 2053) or proliferative diabetic retinopathy (level 61 or above). To evaluate the differences between the study groups, the presence of clinically significant macular oedema (CSMO) was determined, and the fluorescein angiographic findings were graded before the macular thickness measurements were undertaken. CSMO was diagnosed when one or more of the following were detected: retinal thickening involving the centre of the macula or within $500 \mu \mathrm{m}$ of the centre; hard exudate or exudates at or within $500 \mu \mathrm{m}$ of the centre of the macula, when associated with thickening of the adjacent retina; and a zone or zones of retinal thickening one disc area or larger, with any part of the retinal thickening within 1 disc diameter of the centre of the macula. ${ }^{23}$ To evaluate the effect of the breakdown of the blood-retinal barrier on the perifoveal capillary network, the degree of leakage on the fluorescein angiograms at the macula was graded using a modified EDTRS protocol (Table 2). Placing a transparent, size matched, plastic mask with concentric rings over the early phase angiograms, the innermost ring was centred over the foveal avascular zone to grade the leakage from the perifoveal capillary network. Each diabetic patient was clinically examined by one of two retinal specialists (KE, MM) who were masked to the results of the retinal thickness assessment. The angiographic findings were graded by one of us (SY), who did not contribute to the clinical grading portion and was masked to the results of the retinal thickness assessment.

SCANNING RETINAL THICKNESS ANALYSER

The scanning RTA (Talia Technology Ltd, Mevaseret Zion, Israel) is based on the principle of slit lamp biomicroscopy, as previously described. ${ }^{16-18}$ A green helium neon $(534 \mathrm{~nm}$ ) laser slit of approximately $400 \mu \mathrm{W}$ was projected on the retina; the image was scanned by a slit beam and the light passing through the slit was detected by a photodetector and translated into a time dependent electronic signal. The time delay measured between the two maximum values of photodetector output, emanating from the vitreoretinal interface and

Table 2 Grading of fluorescein leakage at the macula

\begin{tabular}{ll}
\hline Level & Definition \\
\hline None & $\begin{array}{l}\text { No dye leakage from perifoveal capillary } \\
\text { network }\end{array}$ \\
Questionable & $\begin{array}{l}\text { Questionable or }<25 \% \text { dye leakage } \\
\text { Focal }\end{array}$ \\
Diffuse & $>66 \%$ but $<66 \%$ dye leakage \\
\hline
\end{tabular}



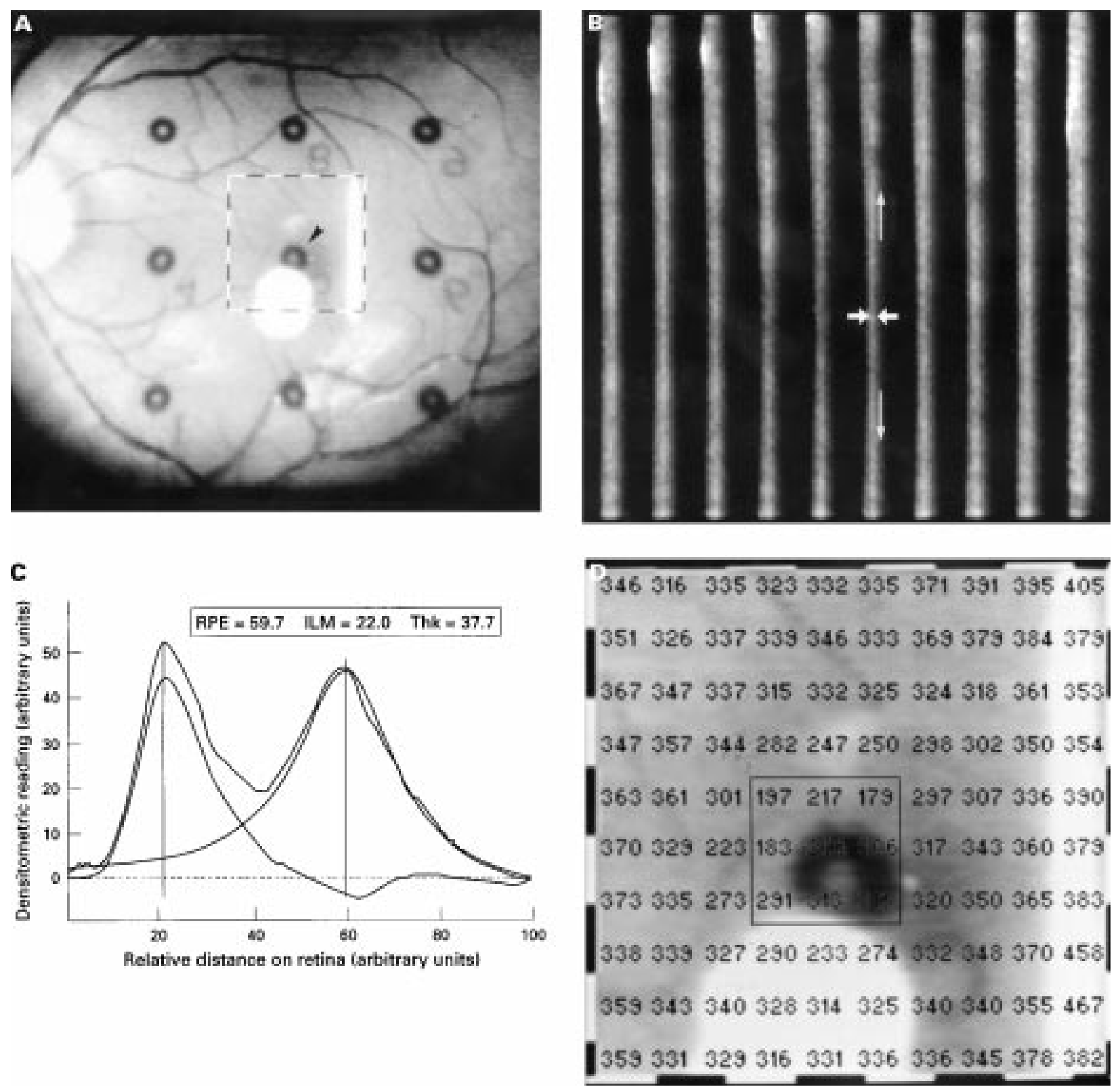

Figure 1 (A) Fundus photograph of a normal left eye in the retinal thickness scan. Nine fixation targets are shown on the posterior fundus. The foveal fixation target is indicated by a black arrowhead, and the scanned retinal area is indicated by the square. (B) $A$ composite of 10 optical section images in a normal left eye covering a retinal area of $2 \times 2 \mathrm{~mm}^{2}$ including the fovea. The left and right arrows indicate the vitreoretinal and chorioretinal interfaces, respectively, at the area of the assumed foveal depression corresponding to the foveal target position. The thin vertical arrows indicate the chorioretinal interfaces. (C) A scan profile of the densitometric reading curve at a spot near the fovea. The distance between the peaks on both sides represent the slit image width that is proportional to the retinal thickness at this point. (D) Numeric grid of the scanned area covering $2 \times 2 \mathrm{~mm}{ }^{2}$. The square indicates the $3 \times 3$ points, which correspond to a $600 \times 600 \mu^{2}$ area, used for the central macular thickness calculation. RPE = retinal pigment epithelium; ILM = internal limiting membrane; Thk = retinal thickness in arbitrary units.

the chorioretinal interface, was proportional to the retinal thickness at the measured location. In the prototype scanning RTA used in the present study, the newest software based on a paradigm analysis algorithm (Version 6.30, Talia Technology Ltd) was developed to analyse the scan images automatically based on this principle.

For the retinal thickness measurement, the pupils were dilated to a minimum of $6 \mathrm{~mm}$ with a mixture of $0.5 \%$ phenylephrine hydrochloride and $0.5 \%$ tropicamide (Mydrin-P, Santen Pharmaceuticals, Osaka, Japan). The subjects were shown a target consisting of a pattern of numbers, and an infrared laser light emitting diode was used for monitoring the fixation (Fig 1A). After activation of a foot pedal, the laser slit scanned a retinal area $2 \times 2$ $\mathrm{mm}^{2}$. Ten slit laser images (Fig 1B) covering this area were obtained within $200 \mathrm{~ms}$. After performing this procedure nine times, an area $6 \times 6 \mathrm{~mm}^{2}$ covering the posterior pole was scanned. In each slit image, 10 points were analysed by an analysis algorithm (Version 6.30, Talia Technology Ltd). At each measured point, the algorithm considered the peaks on both sides of the densitometric reading curve (Fig 1C) to represent the reflections of the outermost and innermost retinal layers. The distance between the two peaks is proportional to the retinal thickness. A relative retinal thickness can be obtained by the following formula; 
$\mathrm{T}=\mathrm{D} \times \mathrm{M} / \mathrm{Sb}$, where $\mathrm{T}=$ relative retinal thickness, $\mathrm{D}=$ the width of the two peaks calculated from the scan profile, $M=$ magnification that changes depending on the refractive error of the individual, and $\mathrm{Sb}=$ stereo base. Thus, the 10 slit images of each scan can be automatically analysed to generate a topographic map in numeric form - that is, 100 points $(10 \times 10)$ of relative retinal thickness values covering a retinal area $2 \times 2 \mathrm{~mm}^{2}$ (Fig 1D). Given the calibration factor based on measurement of the corneal refractive power (dioptres) and refractive error (dioptres) of the study eye, the relative retinal thickness values were automatically converted to absolute micrometre units by the thickness correction software (Talia Technology Ltd).

Before measuring the macular thickness in the diabetic eyes, the intra- and intersession reproducibility of the RTA was evaluated in normal subjects by a single examiner (YO). To determine the intrasession reproducibility, three scans from the centre frame including the macula were evaluated in a single session in each of the normal subjects. One hundred points (10 $\times 10$ ) were evaluated from each scan, and the coefficient of variance (standard deviation/ mean) of the retinal thickness values in all three scans from each subject was calculated for each of the 100 points. To assess the intersession reproducibility, two sessions were carried out at 1 or 2 week intervals; three scans centred on the macula obtained from two different visits were evaluated for each subject. One hundred points $(10 \times 10)$ were evaluated from each scan, and the coefficient of variance (standard deviation/ mean) of the retinal thickness values in all scans of each subject was calculated for each of the 100 points. The intrasession reproducibility also was assessed in the same way in the group of patients with diabetes.

CENTRAL MACULAR THICKNESS MEASUREMENT

Because an area of $2 \times 2 \mathrm{~mm}^{2}$ obtained from a routine fovea centred scan using the RTA is too large to cover the foveal region in EDTRS type regions, ${ }^{23}$ which anatomically corresponds to approximately $500 \mu \mathrm{m}$ in diameter, the average of 100 points $(10 \times 10)$ of the retinal thickness measured from the fovea centred scan (covering an area $2 \times 2 \mathrm{~mm}^{2}$ ) cannot directly represent the central macular thickness. In the central macular thickness assessment, a small area of nine points $(3 \times 3)$ including the fovea, corresponding to an area of $600 \times 600 \mu \mathrm{m}^{2}$, was chosen from a fovea centred scan and defined as the central macula (Fig 1D). Thus, the central macular thickness, which corresponds approximately to the retinal thickness at the foveal region in EDTRS type regions, was calculated as the mean value of the $3 \times 3$ points in the frame acquisition mode obtained from a fovea centred scan image.

STATISTICAL ANALYSIS

Measurement of the central macular thickness was correlated with the visual acuity using linear regression. Visual acuity was measured in each eye using a Snellen visual acuity chart, and then converted to the logarithm of the

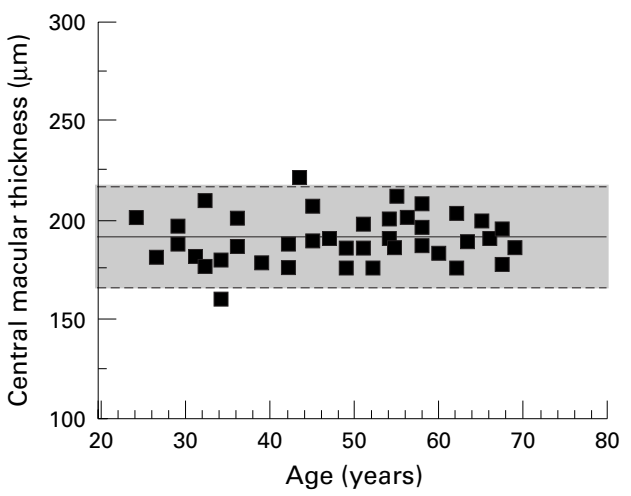

Figure 2 Scattergrams of central macular thickness in normal eyes plotted against the age of the subjects. The central macular thickness did not vary significantly with age $(p>0.99)$, and it was similar for right and left eyes. The solid line represents the mean value that corresponds to 182 $\mu m$, and the two broken lines above and below the solid line indicate the range of $2 S D$. The shaded area represents the normal variation of the central macular thickness measured by the scanning retinal thickness analyser.

minimum angle of resolution (logMAR). In this notation, a score of 0 corresponds to a visual acuity of $20 / 20$ and each doubling of the visual angle increases the score by three units (that is, $20 / 40=3,20 / 50=4$, and $20 / 100=10$ ). To evaluate the differences between the study groups, the unpaired Mann-Whitney $U$ test was used as the non-parametric equivalent of the $t$ test for two independent samples. Spearman's correlation coefficient was calculated to determine the statistical correlation between any two non-normally distributed variables. A $\mathrm{p}$ value smaller than or equal to 0.05 was considered significant.

\section{Results}

REPRODUCIBILITY AND CENTRAL MACULAR

THICKNESS IN NORMAL SUBJECTS

The mean retinal thickness obtained from the $10 \times 10$ measurements of the 40 normal eyes was $312(26) \mu \mathrm{m}$. The mean reproducibility of the scans obtained at the same examination session was $5.2 \%$, which corresponded to approximately plus or minus $16 \mu \mathrm{m}$. The reproducibility of the points obtained from two examinations was plus or minus $6.1 \%$, which corresponds to approximately plus or minus $19 \mu \mathrm{m}$.

The macular thickness at the centre $(3 \times 3$ central points) in the 40 normal eyes is presented as a function of age (Fig 2). The average macular thickness was 182 (16) $\mu \mathrm{m}$. Linear regression of the macular thickness showed no statistically significant correlation between the macular thickness and age (Spearman's correlation coefficient, $r=0.12 ; \mathrm{p}>0.99$ ). The macular thickness was also statistically independent of sex, and no difference was found between left and right eyes.

\section{REPRODUCIBILITY AND CENTRAL MACULAR}

THICKENING IN DIABETIC RETINOPATHY

The mean retinal thickness obtained from the 60 eyes with diabetic retinopathy was 428 (112) $\mu \mathrm{m}$. The mean reproducibility of the scans obtained at the same examination session was plus or minus $6.3 \%$, which corresponded 

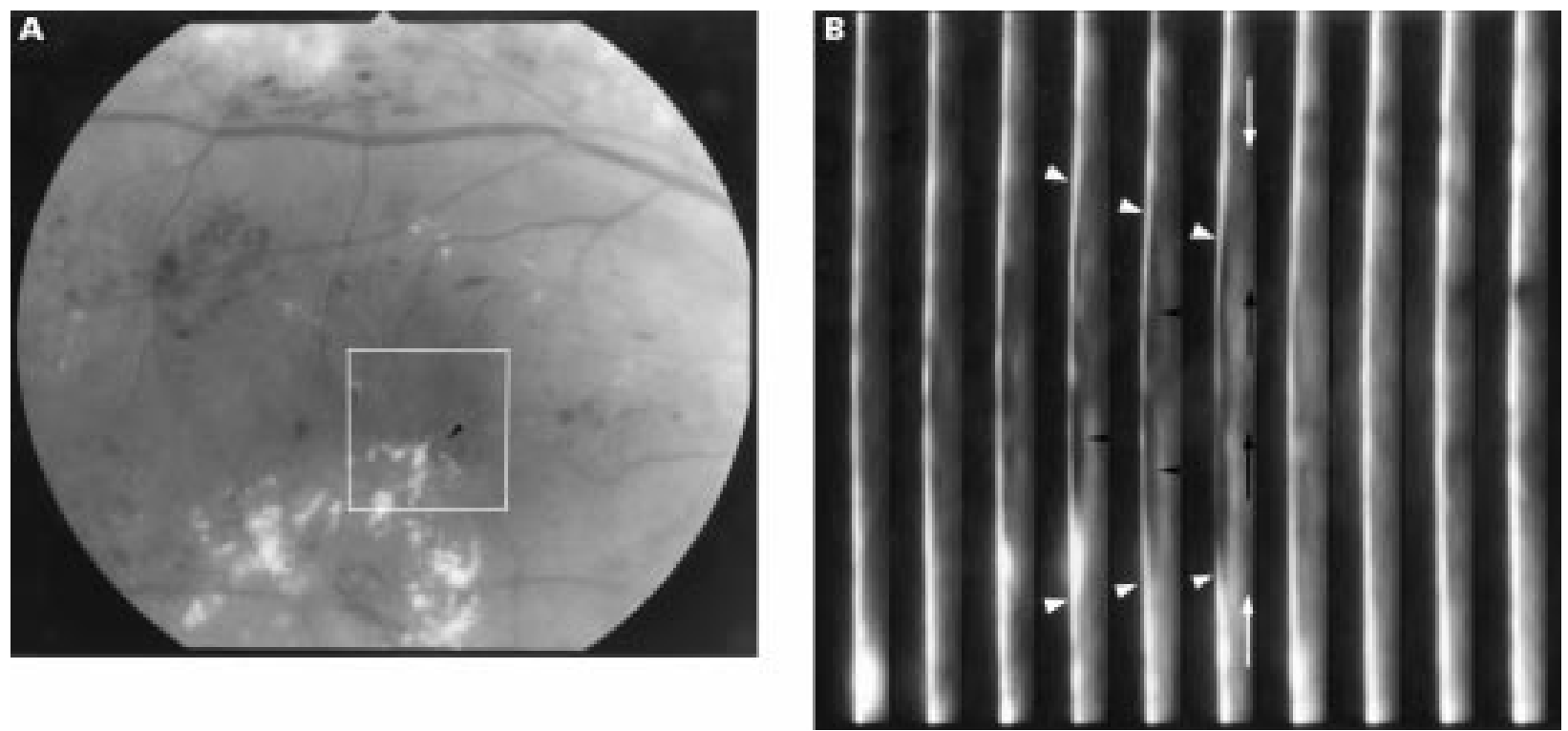

Figure 3 (A) Fundus photograph obtained from the right eye of a patient with localised diabetic macular oedema. Optical sectioning was performed within the area bound by the square. The black arrowhead indicates the fixation target for the central scan, presumably corresponding to the centre of the fovea. (B) A composite of optical section images shows the presence of intraretinal cysts (arrowheads) at the macula that cause elevation of the vitreoretinal interface and loss of a normal foveal depression. The chorioretinal interface (vertical black arrows) is partly obscured because of intraretinal fuid accumulation, but is visualised in the superior and inferior retinal area (vertical white arrows).

to approximately plus or minus $27 \mu \mathrm{m}$. There was no statistically significant difference $(\mathrm{p}=$ 0.24 ) in the intrasession reproducibility between the normal group and the patient group.

Macular thickening in diabetic retinopathy was well delineated on the optical section slit image of the scanning RTA (Figs 3 and 4). The mean central macular thickness of the 60 eyes with diabetic retinopathy was 446 (208) $\mu \mathrm{m}$ (range 156-906 $\mu \mathrm{m}$ ), which was significantly $(p<0.001)$ greater than that of the normal subjects.

RELATION BETWEEN RTA, FLUORESCEIN ANGIOGRAPHY, AND BIOMICROSCOPY

Of the 60 eyes in the retinopathy group, stereo biomicroscopic examination revealed that 36 eyes $(60 \%)$ had CSMO. The visual acuities in eyes with CSMO and in eyes without CSMO varied, respectively, and ranged from $20 / 400$ to $20 / 40$ and $20 / 60$ to $20 / 20$. Fluorescein angiography demonstrated that 21 of 60 eyes $(35 \%)$ had diffuse leakage, and 18 eyes (30\%) had focal leakage at the macular lesions. The results of the central macular thickness measurements using the scanning RTA are summarised in Table 3.

The relation between the distribution of central macular thickness and clinical evaluation with slit lamp biomicroscopy is shown in Figure 5. Although each subset had a wide range of retinal thickness values, the macular thickness was significantly $(p=0.008)$ greater in
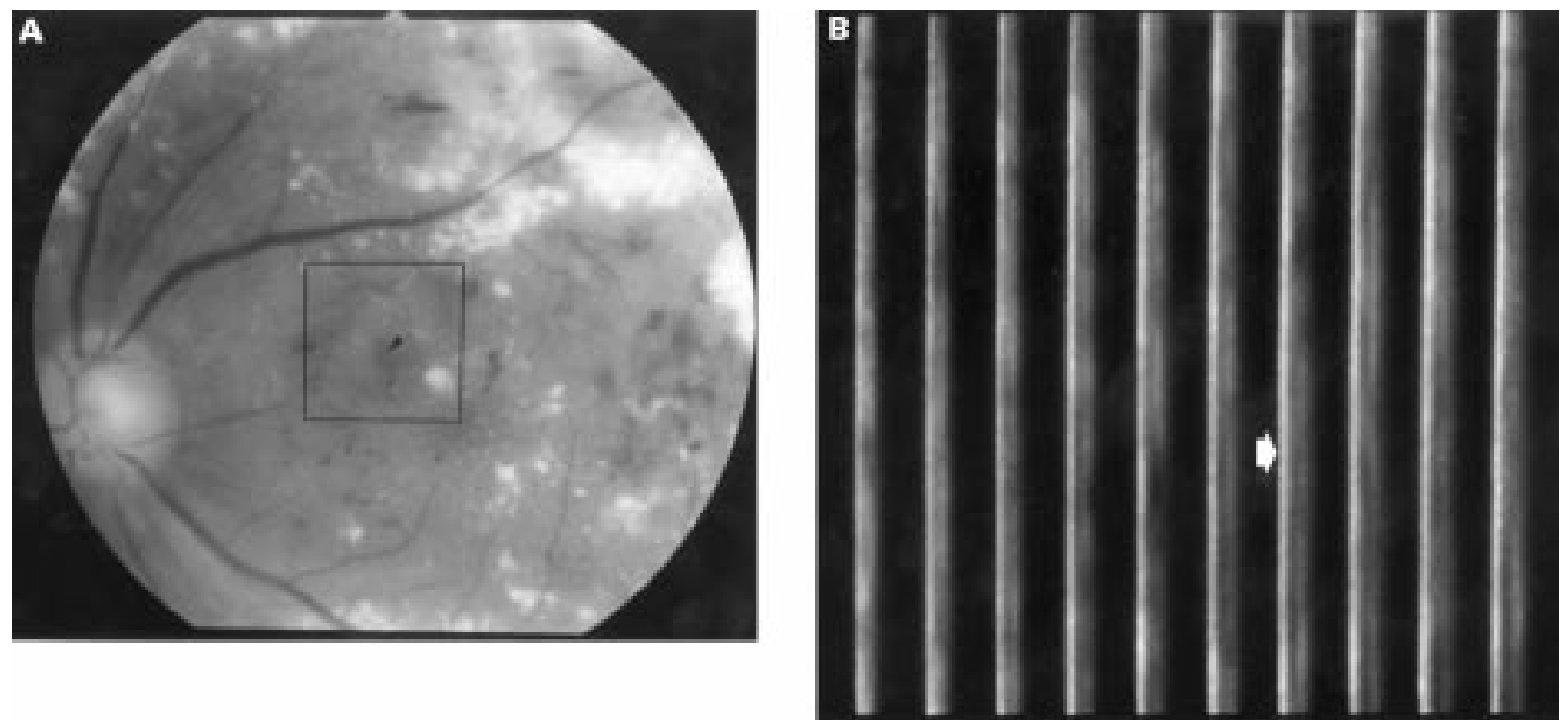

Figure 4 (A) Fundus photograph obtained from the left eye of a patient with diffuse diabetic macular oedema. The square delineates the scanned region demonstrated in the next optical section image. The black arrowhead indicates the fixation target for the central scan, presumably corresponding to the centre of the fovea. (B) Multiple cross sectional scanning image shows the relative elevation of the interretinal surface with gross foveal distortion. The vitreoretinal interface at the centre of the fovea (white arrow) showed an absent foveal depression. 


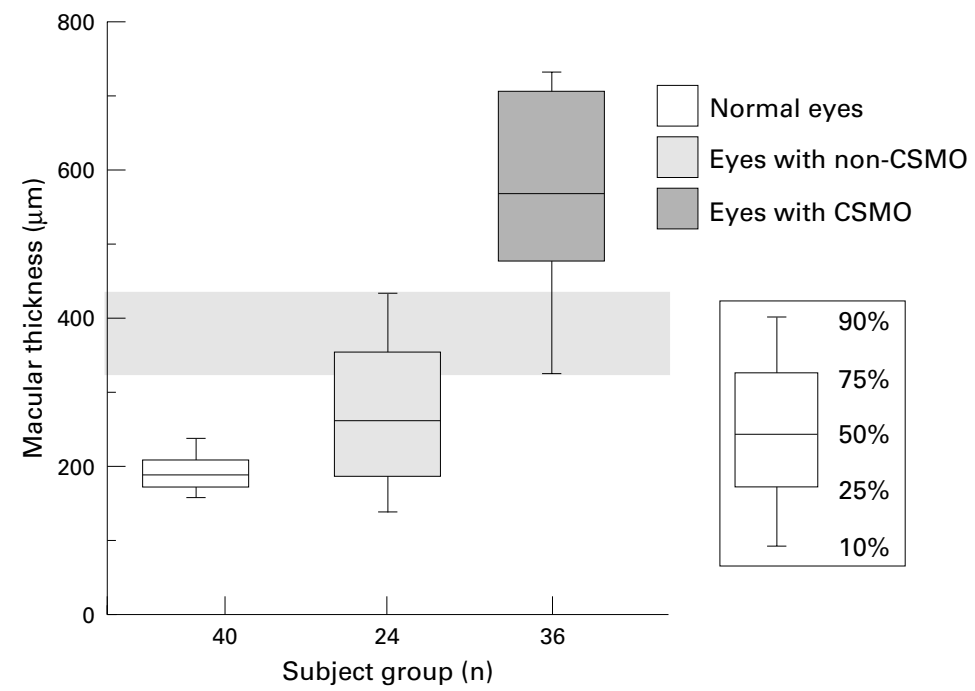

Figure 5 Box plots of the central macular thickness in the three groups determined by using the scanning retinal thickness analyser. The macular thickness is significantly greater $(p=0.008)$ in the eyes with clinically significant macular oedema (CSMO) than in the eyes without CSMO. The retinal thicknesses in the eyes with non-CSMO were significantly $(p=0.019)$ greater than in the normal control eyes. The boxes include $50 \%$ of the measured values (25\% and $75 \%$ percentiles) and demonstrate the position of the median (horizontal line). The shaded area indicates the thickness range in which CSMO and non-CSMO diagnoses overlapped (range 320-420 $\mathrm{m}$ ). CSMO = clinically significant macular oedema .

the eyes with CSMO than in those without CSMO, which was significantly $(p=0.019)$ greater than in normal control subjects.

Regarding the relation between macular thickening and the grading of fluorescein leakage on angiograms, greater degrees of fluorescein leakage are usually considered to be associated with greater central macular thickness. However, only eyes with diffuse fluorescein leakage had statistically significant $(p=0.022)$ macular thickening compared with the normal subjects. Figure 6 shows the relation between the distribution of central macular thickness and fluorescein leakage. The macular thickness was significantly greater in eyes with diffuse fluorescein leakage than in the eyes in any of the other subsets. However, there were no significant differences between eyes with focal fluorescein leakage and each of the other two subsets as a result of a wide range of thickness values in each subgroup.

\section{RELATION BETWEEN CENTRAL MACULAR}

THICKNESS AND VISUAL ACUITY

The central macular thickness was significantly correlated with visual acuity converted to a

Table 3 Average macular thicknesses in the study groups

\begin{tabular}{|c|c|c|c|}
\hline Group & $\begin{array}{l}\text { Central macular } \\
\text { thickness }(\mu m) t \\
\text { Mean }(S D)\end{array}$ & $\begin{array}{l}\text { Range } \\
(\mu \mathrm{m})\end{array}$ & $p$ Value ${ }^{\star}$ \\
\hline Normal eyes $(n=40)$ & $182(16)$ & $164-221$ & \\
\hline \multicolumn{4}{|c|}{ Eyes with diabetic retinopathy $(n=60)$} \\
\hline \multicolumn{4}{|c|}{ CSMO detection by slit lamp biomicroscopy } \\
\hline non-CSMO $(\mathrm{n}=24)$ & $283(116)$ & $156-423$ & 0.019 \\
\hline $\operatorname{CSMO}(n=36)$ & $564(168)$ & $278-906$ & $<0.001$ \\
\hline \multicolumn{4}{|c|}{ Leakage grading using fluorescein angiography } \\
\hline None $(n=10)$ & $187(76)$ & $156-328$ & 0.597 \\
\hline Questionable leakage $(n=11)$ & $191(151)$ & $146-388$ & 0.678 \\
\hline Focal leakage $(n=18)$ & $320(146)$ & $181-562$ & 0.069 \\
\hline Diffuse leakage $(n=21)$ & $552(166)$ & $324-906$ & 0.022 \\
\hline
\end{tabular}

${ }^{\star}$ Central macular thickness compared with normal subjects. Statistical analysis was done using the unpaired Mann-Whitney U test.

†Central macular thickness was measured using the scanning retinal thickness analyser.

$\mathrm{CSMO}=$ clinically significant macular oedema.
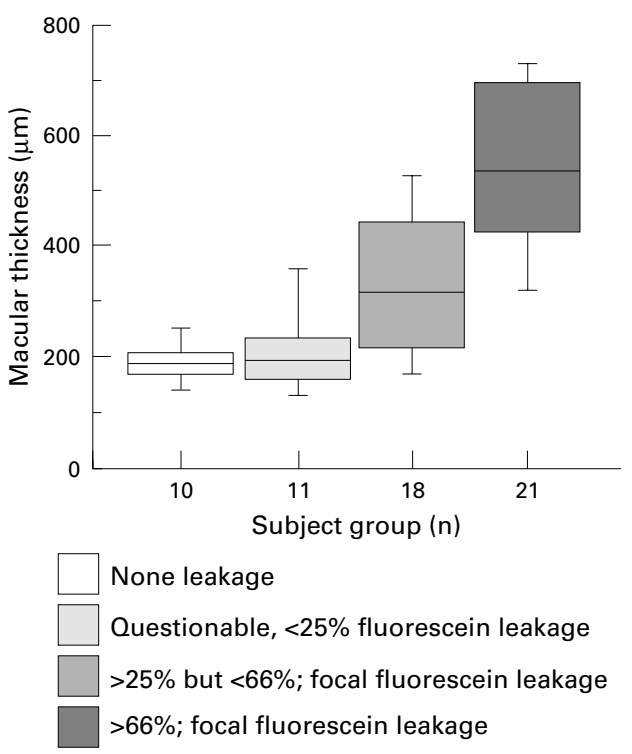

Figure 6 Box plots of central macular thickness determined using the scanning retinal thickness analyser in four subsets grading by fluorescein angiography. The macular thickness is significantly $(p=0.022)$ greater in eyes with diffuse fluorescein leakage than in eyes with focal fluorescein leakage. However, no significant differences in macular thickness are seen between eyes with focal fluorescein leakage and each of the other two subsets as a result of a wide range of thickness values in each subgroup. The positions of the medians (solid line within the boxes) illustrate the increasing macular thickness in eyes with increasing degrees of fluorescein leakage.

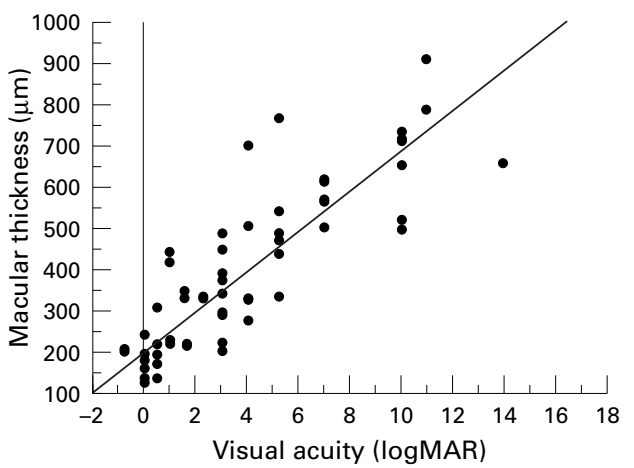

Figure 7 The relation between central macular thickness and visual acuity in eyes with diabetic retinopathy. The visual acuity is reported as the logarithm of the minimum angle of resolution $(\log M A R)$. Linear regression demonstrates a good correlation $\left(n=60 ; r^{2}=0.76 ; p<0.001\right)$ between the macular thickness and visual acuity.

logMAR scale (Spearman's correlation coefficient, $r=0.87 ; \mathrm{p}<0.001)$ in eyes with diabetic retinopathy (Fig 7). The equation for the linear correlation was based on the following formula: macular thickness $(\mu \mathrm{m})=195.8+48.7 \times$ $\log \operatorname{MAR}\left(r^{2}=0.76\right)$.

\section{Discussion}

In the present study, the results indicate that for quantifying retinal thickness the scanning RTA is highly reproducible and has low intraand intersession variability. Under standard conditions, the standard deviation of plus or minus $26 \mu \mathrm{m}$ indicates that the scanning RTA detects retinal thickening of more than approximately $50 \mu \mathrm{m}$. The intrasession reproducibility of plus or minus $5.2 \%$ and the intersession reproducibility of plus or minus $6.1 \%$, 
which correspond to approximately 16 and 19 $\mu \mathrm{m}$, respectively, suggest that the scanning RTA can clinically screen changes in retinal thickening. The intrasession reproducibility in the patient group (plus or minus 6.3\%) indicates that the scanning RTA also could detect the retinal thickness in eyes with diabetic retinopathy enrolled in the present study.

The mean central macular thickness in normal eyes measured in the present study was $182 \mu \mathrm{m}$, which is comparable to the results reported in two previous studies. ${ }^{16}{ }^{18}$ The absolute values of macular thickness quantified by the scanning RTA are still uncertain because a comparison between the in vivo retinal thickness measurements and histological analysis has not been conducted. However, for the present, central macular thicknesses ranging from 150 to $214 \mu \mathrm{m}$ (within twice the standard deviation of the average normal value) can be considered to be within the normal variations of the RTA measurement.

Data from the normal subjects in the present study showed slight variability in macular thickness. However, no statistically significant relation between macular thickness and aging was found. Figure 2 shows that the central macular thickness is independent of patient age. This result differs from the results reported by Landau and associates, ${ }^{18}$ in which the foveola thickness increased with age. Additional studies with more subjects are required to resolve this discrepancy.

A cross sectional study reported that macular oedema in various retinal disorders has no direct association with visual acuity. ${ }^{6}$ However, a previously published study evaluated macular thickening using only qualitative methods such as slit lamp biomicroscopic examination, fundus photography, or fluorescein angiography. With a quantitative measurement of the retinal thickness, foveal thickening was strongly associated with decreased visual acuity. ${ }^{19}$ In the present study, the central macular thickness measured using the RTA was found to be linearly correlated with visual acuity expressed on a logMAR scale $\left(r^{2}=0.76\right)$, which agrees with the results of studies that measured the macular thickness in retinal diseases using optical coherence tomography. ${ }^{2024}$

Routine slit lamp biomicroscopy can provide information that is useful in the clinical diagnosis of macular oedema. However, the actual macular thickening, particularly in the early stage of macular oedema, is hard to estimate using slit lamp biomicroscopy. Shahidi and associates reported that clinical examination using slit lamp biomicroscopy permits the detection of retinal thickening only when it is more than $60 \%$ of the normal retinal thickness. ${ }^{19}$ In the present study, 24 eyes $(40 \%)$ with various degrees of diabetic retinopathy were clinically diagnosed without CSMO. However, the macular thickness was significantly greater $(p=0.019)$ in these eyes than in normal control subjects. When CSMO was found, the central macular thickness markedly increased to more than $350 \mu \mathrm{m}$ based on our results. Slit lamp biomicroscopy cannot iden- tify mild or localised macular thickening. Because biomicroscopy grading is based on a distinction of the local differences rather than comparisons with absolute normal values, it also is limited in detecting diffuse oedema as a total elevation of the macula. By quantitatively measuring the actual thickness, scanning RTA can more reliably detect changes in central macular thickness before CSMO develops.

Fluorescein angiography is traditionally used to assess retinal vascular permeability in patients with diabetic retinopathy. The degree of fluorescein leakage at the macula was generally considered to be associated with retinal thickening. However, our results show that this qualitative method cannot reliably detect changes in macular thickening. The wide variations in thickness values in each subgroup led us to propose that the degree of fluorescein leakage is not directly correlated with retinal thickening. Fluorescein leakage, which physiologically indicates the breakdown of the bloodretinal barrier, is not always accompanied by appreciable fluid accumulation in the retina. ${ }^{5}$ In a subclinical stage, slight dye leakage on fluorescein angiography could appear without retinal thickening because the balance between the vascular permeability and the ability of the retinal pigment epithelium to reabsorb fluid has been maintained. As retinopathy progresses, this balance is disturbed, resulting in fluid accumulation and macular oedema. ${ }^{8}$ Thus, fluorescein angiography is useful for evaluating the severity of the dysfunction in the blood-retinal barrier, but it does not reliably quantify the degree of fluid accumulation in the retina. The scanning RTA is superior to these conventional methods for detecting macular thickening because of multiple cross sectional imaging and the direct measurement of retinal thickness.

In our clinical experience with scanning RTA, one of the chief advantages of this tool over other quantitative instrument is its short acquisition time. Within the $200 \mathrm{~ms}$ required for scanning, patients have no discomfort caused by prolonged light exposure, the need for steady ocular fixation, or lack of blinking. Furthermore, multiple cross sectional retinal imaging, which generates three dimensional reconstruction of the retina, may contribute to a better understanding of retinal elevation. However, we are also aware of sources of error attributable to the instrument that can explain the variability observed in the present study. The most common reasons for inadequate image quality are the irregular reflections from hard exudates accumulated in the retina and the interference of the media opacities, such as severe cataract or vitreous opacity, with the scanning of the laser. Massive intraretinal exudation can prevent the relatively short wave length of the green helium neon $(534 \mathrm{~nm})$ from obtaining an accurate reflection from the chorioretinal interface, resulting in unclear laser slit images. Therefore, we still believe that this prototype RTA is limited in its ability to measure the central macular thickness, particularly in patients with extensive hard exudates that accumulated at the fovea, and thus 
we excluded them from the present study. However, because this type of exudation is rarely found in the early stage of diabetic retinopathy, most types and various degrees of diabetic retinopathy could be effectively evaluated with this novel instrument as we showed in the present study.

In conclusion, this study suggests that the scanning RTA, which provides multiple cross sectional visualisation of the retinal, is useful to measure quantitatively macular thickening in diabetic retinopathy. Although we do not recommend that scanning RTA replace any other conventional diagnostic tool used to detect macular oedema because of its limitations, we believe that scanning RTA may contribute to early, accurate diagnosis of macular thickening in diabetic retinopathy. However, further randomised studies with large populations are required to evaluate this tool for longitudinal monitoring of the disease prognosis and treatment efficacy.

The authors have no proprietary interests in any of the materials used in this study.

Presented in part at the Annual Meeting of the Association for Research in Vision and Ophthalmology, Fort Lauderdale, Florida, May 1997.

1 Ferris F, Patz A. Macular edema: a complication of diabetic retinopathy. Surv Ophthalmol 1984;28:452-61.

2 Early Treatment Diabetic Retinopathy Study Research Group. Photocoagulation for diabetic macular edema: Early Treatment Diabetic Retinopathy Study report no 1. Arch Ophthalmol 1985;103:1796-806.

3 Conway MD, Olk RJ. Diabetic maculopathies: diagnosis and treatment. Ophthalmol Clin N Am 1993;6:213-30.

4 Gass JDM. Pathophysiologic and histopathologic bases for interpretation of fluorescein angiography. In: Gass JDM. interpretation of fluorescein angiography. In: Gass JDM. Stereoscopic atlas of macular diseases: diagno
3rd ed. St Louis: CV Mosby, 1987:19-42

5 Shatz H. Fluorescein angiography: basic principles and interpretation. In: Ryan SJ. Retina. St Louis: CV Mosby, 1989:3-77

6 Smith RT, Lee CM, Charles HC, et al. Quantification of diabetic macular edema. Arch Ophthalmol 1987;105:21822.

7 Onishi Y, Fujikawa K, Ishibashi T, et al. Capillary blood flow velocity measurements in cystoid macular edema with scanning laser ophthalmoscope. Am f Ophthalmol 1994; 117:24-9.
8 Arend O, Remky A, Elsner AE, et al. Quantification of cystoid changes in diabetic maculopathy. Invest Ophthalmol Vis Sci 1995;36:608-13.

9 Moss SE, Klein R, Kessler SD, et al. Comparison between ophthalmoscopy and fundus photography in determining severity of diabetic retinopathy. Ophthalmology 1985;92:62-

10 Kinyoun J, Barton F, Fisher M, et al. Detection of diabetic macular edema: ophthalmoscopy versus photography: Early Treatment Diabetic Retinopathy Study report no 5. Ophthalmology 1989;96:746-51.

11 Kiri A, Dyer DS, Bressler NM, et al. Detection of diabetic macular edema: Nidek 3Dx stereophotography compared with fundus biomicroscopy. Am f Ophthalmol 1996;122: 654-62.

12 Shahidi M, Zeimer R, Mori M. Topography of the retinal thickness in normal subjects. Ophthalmology 1990;97: $1120-4$.

13 Hee MR, Izatt JA, Swanson EA, et al. Optical coherence tomography of the human retina. Arch Ophthalmol 1995;113:325-32.

14 Hudson C, Flanagan JG, Turner GS, et al. Scanning laser tomography $\mathrm{Z}$ profile signal width as an objective index of tomography $Z$ profile signal width as an objective index of 30 .

15 Zeimer R, Mori MT, Khoobehi B. Feasibility test of a new method to measure retinal thickness noninvasively. Invest Ophthalmol Vis Sci 1989;30:2099-105.

16 Zeimer R, Shahidi M, Mori MT, et al. A new method for rapid mapping of the retinal thickness at the posterior pole. Invest Ophthalmol Vis Sci 1996;37:1994-2001.

17 Asrani S, Zeimer R, Goldberg MF, et al. Application of rapid scanning retinal thickness analysis in retinal diseases. Ophthalmology 1997;104:1145-51.

18 Landau D, Schneidman EM, Jacobovitz T, et al. Quantitative in vivo retinal thickness measurement in healthy subjects. Ophthalmology 1997;104:639-42.

19 Shahidi M, Ogura Y, Blair NP, et al. Retinal thickness analysis for quantitative assessment of diabetic macular edema. Arch Ophthalmol 1991;109:1115-19.

20 Hee MR, Puliafito CA, Fuker JS, et al. Topography of diabetic macular edema with optical coherence tomography. Ophthalmology 1998;105:360-70.

21 Zambarakji HJ, Amoaku WM, Vernon SA. Volumetric analysis of early macular edema with the Heidelberg retina tomograph in diabetic retinopathy. Ophthalmology 1998; 105:1051-9.

22 Early Treatment Diabetic Retinopathy Study Research Group. Fundus photographic risk factors for progression of diabetic retinopathy: Early Treatment Diabetic Retinopathy Study report no 12. Ophthalmology 1991;98:823-33.

23 Early Treatment Diabetic Retinopathy Study Research Group. Treatment techniques and clinical guidelines for photocoagulation of diabetic macular edema: Early Treatment Diabetic Retinopathy Study report no 2. Ophthalmology 1987;94:761-74.

24 Wilkins JR, Puliafito CA, Hee MR, et al. Characterization of epiretinal membranes using optical coherence tomography. Ophthalmology 1995;103:2142-51. 